\title{
One-Dimensional Ising Chain with Competing Interactions: Exact Results and Connection with Other Statistical Models
}

\author{
S. Redner ${ }^{1}$
}

Received March 20, 1980

We study the ground state properties of a one-dimensional Ising chain with a nearest-neighbor ferromagnetic interaction $J_{1}$, and a $k$ th neighbor antiferromagnetic interaction $J_{k}$. When $J_{k} / J_{1}=-1 / k$, there exists a highly degenerate ground state with a residual entropy per spin. For the finite chain with free boundary conditions, we calculate the degeneracy of this state exactly, and find that it is proportional to the $(N+k-1)$ th term in a generalized Fibonacci sequence defined by, $F_{N}^{(k)}=F_{N-1}^{(k)}+F_{N-k}^{(k)}$. In addition, we show that this one-dimensional model is closely related to the following problems: (a) a fully frustrated two-dimensional Ising system with a periodic arrangement of nearestneighbor ferro- and antiferromagnetic bonds, (b) close-packing of dimers on a ladder, a $2 \times \infty$ strip of the square lattice, and (c) "directed" self-avoiding walks on finite lattice strips.

KEY WORDS: One-dimensional Ising chain; competing interactions; ground state degeneracy; Fibonacci sequence; close packing of dimers; directed self-avoiding walks.

\section{INTRODUCTION}

Consider a one-dimensional Ising chain with nearest- and $k$ th-neighbor interactions $J_{1}$ and $J_{k}$, respectively. The Hamiltonian for this system is

$$
\mathscr{H}=-J_{1} \sum_{i} s_{i} s_{i+1}-J_{k} \sum_{i} s_{i} s_{i+k}
$$

Work partially supported by grants from AFOSR and ARO.

${ }^{1}$ Center for Polymer Studies and Department of Physics, Boston University, Boston, Massachusetts 02215 . 
For $J_{1}>0$ and $J_{k}<0$, the two interactions compete in determining the ground state of the system. This competition effect ${ }^{(1)}$ is of current interest because of its possible relevance as the mechanism for both spin glass behavior ${ }^{2}$ and helical ordering in many magnetic systems.

To describe helical order in a $d$-dimensional system, one can consider a chain of $(d-1)$-dimensional ferromagnetically coupled layers, with interactions along the chain given by Eq. (1). Generally, the case of nearest- and second-neighbor interactions $(k=2)$ has been studied most often. The resulting model, which we call the RS model, ${ }^{(3)}$ was first considered by Enz $^{(4)}$ and Elliott ${ }^{(5)}$ to describe the magnetism of the rare earths. Recent investigations of this model have focused on the Lifshitz point, where there is a coexistence of disordered, ferromagnetic, and helical phases, ${ }^{(3,6,7)}$ and on the striking features at low temperatures. ${ }^{(8,9,10)}$ Here an infinite sequence of commensurate phases occurs as $J_{2} / J_{1}$ varies, and as $T \rightarrow 0$ these phases coalesce into a single infinitely degenerate phase at $J_{2} / J_{1}=-1 / 2$.

This degenerate phase exhibits some very striking features; therefore we have studied the one-dimensional system where we can obtain exact results. In the thermodynamic limit, the exact solution for the $k=2$ case (RS model) has been found previously, ${ }^{(11)}$ but we are able to calculate exactly the ground state properties for both the finite and infinite chain for all values of $k$. We find that when $J_{k} / J_{1}=-1 / k$, the ground state is highly degenerate for any number of spins $N \geqslant 2 k$. Moreover, for finite $N$, we can express this degeneracy in terms of a simple generalization of the Fibonacci sequence. From the asymptotic behavior of this sequence, we derive very simply a closed-form expression for the entropy in the thermodynamic limit. In addition, we find that the Ising chain can be mapped onto several apparently different statistical models, leading to further exact results.

\section{THE GROUND STATE AND ITS DEGENERACY}

To begin, we consider the nature of ground state for $J_{1}>0$ and varying $J_{k}$ (see Fig. 1). For $J_{k}<0$ but sufficiently small, ferromagnetism occurs. For $J_{k}<0$ but large, every $k$ th spin orders antiferromagnetically, and to obtain the ground state, the energy in the remaining ferromagnetic bonds must be a minimum. This happens when there are $k$ spins $\uparrow$, then $k$ spins $\downarrow$, etc., and we denote this phase as $\langle k\rangle^{j}$.

Now consider the case $J_{k} / J_{1}=-1 / k$. In an infinite chain, the energy per spin of the ferromagnetic and $\langle k\rangle$ phases, $E_{\text {ferro }}=-J_{1}-J_{k}$ and $E_{\langle k\rangle}=(2 / k) J_{k}+[(k-2) / k]\left(-J_{1}+J_{k}\right)$, respectively, are equal. Thus the

\footnotetext{
${ }^{2}$ We use the notation of Ref. 8 .
} 


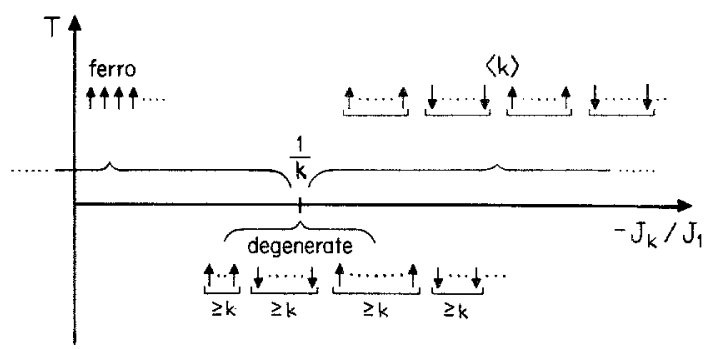

Fig. 1. The zero temperature phase diagram for the system described by the model Hamiltonian of Eq. (1). The three ordered phases that occur are indicated schematically.

ground state consists of a random mixture of ferromagnetic and $\langle k\rangle$ phases, leading to a large degeneracy. We can describe this degenerate phase by the following simple picture: Starting with the ferromagnetic phase we may introduce a domain wall by flipping all the spins to one side of a given spin. Any number of such walls can be successively created with no energy cost as long as the walls are $\geqslant k$ lattice spacings apart (and also $\geqslant k$ lattice spacings from the end of the chain with free boundaries). Therefore this phase has domains of $\geqslant k$ spins $\uparrow$, followed by domains of $\geqslant k$ spins $\downarrow$, etc., as indicated in Fig. 1 .

To find the degeneracy associated with this phase, we first describe our calculation for the RS model $(k=2)$, and then outline a simple generalization for arbitrary $k$. In what follows we employ free boundary conditions, and we also assume that the number of spins $N$ in the chain is $\geqslant 2 k$. (For $N<2 k$, the ground state is only twofold degenerate.)

Let $a_{N}$ be the total number of degenerate ground state spin configurations of a chain of $N$ spins. This number equals $b_{N}$, the number of configurations in which the last two spins are parallel, plus $c_{N}$, the number of configurations in which the last two spins are antiparallel. Now $b_{N}$ can be obtained by adding an additional spin parallel to the $(N-1)$ th spin for any configuration in $b_{N-1}$ and $c_{N-1}$. Hence

$$
b_{N}=b_{N-1}+c_{N-1}=a_{N-1}
$$

We can obtain $c_{N}$ by adding a spin antiparallel to the $(N-1)$ th spin for any configuration in $b_{N-1}$. Therefore

$$
c_{N}=b_{N-1}
$$

(Notice that we cannot add an antiparallel spin to a configuration in $c_{N}$ because this would lead to an energetically unfavored domain of a single spin.) Combining (2a) and (2b) yields

$$
a_{N}=b_{N}+c_{N}=a_{N-1}+a_{N-2}
$$


This is just the recursion relation for the Fibonacci sequence defined by $F_{N}^{(2)}=F_{N-1}^{(2)}+F_{N-2}^{(2)}$, and $F_{1}^{(2)}=F_{2}^{(2)}=1$. We therefore obtain $a_{N}=$ $2 F_{N+1}^{(2)}$.

For arbitrary $k$, we may add the $N$ th spin parallel to the $(N-1)$ th spin for any configuration in $a_{N-1}$. On the other hand if we add the $N$ th spin antiparallel to the previous spin, then at least spins $N-1$ through $N-k$ must all be antiparallel to the $N$ th spin. Because of the determinancy in these $k$ spins, any such state of $N-1$ spins corresponds uniquely to a state in $a_{N-k}$. Hence we find

$$
a_{N}=a_{N-1}+a_{N-k}
$$

This recursion relation defines a "generalized" Fibonacci sequence $F_{N}^{(k)}=$ $F_{N-1}^{(k)}+F_{N-k}^{(k)}$, with $F_{1}^{(k)}=F_{2}^{(k)}=\cdots=F_{k}^{(k)}=1$. From this we obtain $a_{N}=2 F_{N+k-1}^{(k)}$.

It is interesting to examine the $k$ dependence of the entropy per spin $S^{(k)}$ in the thermodynamic limit. To obtain this limit, we first note that the Fibonacci sequence becomes purely geometric as $N \rightarrow \infty$. This follows by writing $F_{N-1}^{(k)}, F_{N-2}^{(k)}, \ldots, F_{N-k}^{(k)}$ as the elements of a vector $\mathbf{v}_{N-1}$, and then writing the $k \times k$ transfer matrix transforming $\mathbf{v}_{N-1}$ to $\mathbf{v}_{N}$. This matrix has only one eigenvalue $>1$, and this gives the multiple in the geometric sequence. Equivalently we can find this eigenvalue more easily by using $F_{N}^{(k)} \sim x^{N}$ in the defining relation for the Fibonacci sequence. This yields the characteristic equation, $x^{N}=x^{N-1}+x^{N-k}$, or $x^{k}=x^{k-1}+1$. From the largest root of this equation, we find $S^{(2)}=\log [(1+\sqrt{5}) / 2]$. This agrees with the entropy found from taking the exact expression for the free energy, ${ }^{(11)}$ and evaluating it at $J_{2} / J_{1}=-1 / 2$. For $k>2$, the largest root of the characteristic equation yields $S^{(3)}=\log (1.4665 \ldots), S^{(4)}=$ $\log (1.3802 \ldots), S^{(5)}=\log (1.3247 \ldots)$, etc. As $k \rightarrow \infty$, the spin domains become infinitely large, and this corresponds to the entropy approaching 0 . In this limit, the degenerate and $\langle k\rangle$ phases become identical to the ferromagnetic phase.

\section{CONNECTION WITH OTHER STATISTICAL MODELS}

In addition to elucidating the striking features of the degenerate phase, we show that the Ising chain with competing interactions has close connections with several apparently diverse problems.

(a) A fully frustrated two-dimensional Ising model: The piled up dominoes (PUD) model introduced by André et al. ${ }^{(12)}$ is a two-dimensional Ising model with a periodic arrangement of nearest-neighbor ferro- and antiferromagnetic interactions of different strengths $J$ and $J^{\prime}$, respectively (see Fig. 2a). Every elementary plaquette consists of an odd number of 


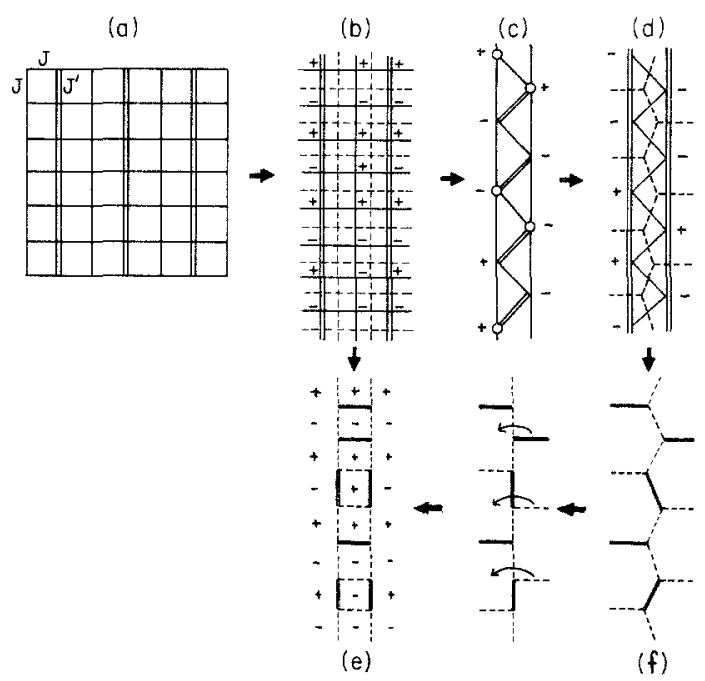

Fig. 2. (a) The PUD model: The single and double bonds represent ferromagnetic interactions $J$, and antiferromagnetic interactions $J^{\prime}$, respectively. (b) A section of the PUD model with the dual lattice shown dashed. The + and - signs indicate the spins in a typical ground state for $\left|J^{\prime}\right|>J$. The antiferromagnetic vertical chains order "in phase," in one of two possible states. On the other hand, there is a large degeneracy associated with the possible states of the vertical ferromagnetic chains. (c) Under the transformation $s_{i} \rightarrow t_{i} t_{i+1}$, the vertical ferromagnetic chain in (b) maps into a chain with alternating nearest-neighbor interactions (zig-zags), and ferromagnetic second-neighbor interactions (vertical). The + and - signs now indicate the signs of the new spin variables $t_{i}$. These are defined only up to an overall sign; consequently there is a two-to-one correspondence between the ground states of the RS model and a ferromagnetic chain in the PUD model. The open circles indicate the spins to be redefined in order to map the Ising chain to the RS model through a gauge transformation. (d) The RS model, with ferromagnetic nearest-neighbor interactions, and antiferromagnetic second-neighbor interactions. The + and - signs indicate the spin states obtained after the gauge transformation from (c). Shown dashed is the dual lattice. (e) The dual ladder of the PUD model and the spins states of the original lattice from (b). The close-packed dimer configurations that are derived from this spin state are indicated by the full lines. (f) The dual of the RS model with the close-packed dimer configuration corresponding to the spin state of (d). From this dimer configuration we can obtain the geometrically equivalent configuration (e) as follows: First we "straighten out" the dual lattice, and then slide alternate horizontal bonds to one side of the vertical chain. Finally, we add a second vertical dimer chain identical to the first one to obtain the dual ladder in the dimer state (e).

antiferromagnetic bonds, hence each plaquette is frustrated in the ground state. ${ }^{(1)}$ This model is interesting because it is equivalent to a spin-glass-like model in which the $J$ and $J^{\prime}$ bonds can be rearranged in an apparently random way by a gauge transformation. ${ }^{(13)}$

In the ground state, the vertical chains of antiferromagnetic bonds order "in phase" when $\left|J^{\prime}\right|>J$ (see Fig. 2b). Consequently the system is 
essentially one dimensional because each vertical ferromagnetic chain feels only a staggered magnetic field of strength $2 J$ in addition to the nearestneighbor interaction. Thus we may write the following effective Hamiltonian for the ferromagnetic chains:

$$
\mathcal{K}_{\mathrm{eff}}=-J \sum_{i} s_{i} s_{i+1}-2 J \sum_{i}(-1)^{i} s_{i}
$$

We can show that this system is equivalent to the RS model by first using the well-known transformation $s_{i} \rightarrow t_{i} t_{i+1}$ to rewrite (3a) as

$$
\mathcal{K}_{\mathrm{eff}}^{\prime}=-J \sum_{i} t_{i} t_{i+2}-2 J \sum_{i}(-1)^{i} t_{i} t_{i+1}
$$

Now we have a spin chain with alternating ferro- and antiferromagnetic nearest-neighbor interactions, and a second-neighbor ferromagnetic interaction as shown in Fig. 2c. Next we perform the gauge transformation indicated in the figure. We reverse the signs of the spins at the circled sites, and reverse the signs of the bonds incident on these spins. Thus all nearest-neighbor bonds become ferromagnetic, and all second-neighbor bonds become antiferromagnetic, and we have obtained the RS model when $J_{2} / J_{1}=-1 / 2$ (see Fig. 2d).

(b) Dimer statistics: Another way to understand the equivalence of the PUD and RS models, is to map the degenerate spin ground states into close-packed dimer configurations on the respective dual lattices. That is, we place a dimer on a dual bond which crosses a frustrated bond on the original lattice (Fig. 2e). In the ground state of the PUD model, exactly one bond per plaquette is frustrated; this implies that the dimers must be close packed on the dual. ${ }^{(12)}$ Moreover, because the antiferromagnetic chains are all "in phase," frustrated horizontal bonds occur in pairs. Consequently, the vertical dimers between any two antiferromagnetic chains must also occur in pairs. Thus evaluating the ground state entropy of the PUD model is equivalent to counting the number of corresponding close-packed dimer configurations on a "ladder," a $2 \times \infty$ strip of the square lattice.

Now consider the RS model: The minimum energy spin states also map to close-packed dimer configurations on the dual, a strip of the hexagonal lattice (Fig. 2f). By following the simple geometric manipulations indicated in the lower portion of Fig. 2, we deduce that counting the close-packed dimer configurations for the two problems are identical.

(c) "Directed" self-avoiding walks: We define "directed" self-avoiding walks (SAW) as random walks which may visit a particular lattice site only once, and which have the additional constraint that steps in one direction are prohibited (see Fig. 3a). To see the connection between such walks on a ladder and the Ising chain, we imagine placing a spin up at each visited site on the upper edge of the ladder, and vice versa on the lower edge. Upon 

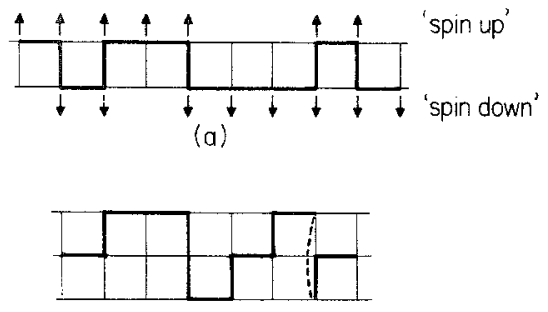

(b)

Fig. 3. (a) A directed SAW on the ladder of width two lattice spacings. Steps to the left are prohibited. At each site, we place a spin up if the walk passes through a site on the upper edge of the ladder, and vice versa for the lower edge. As the walk proceeds we trace out one of the spin configurations in the degenerate ground state of the RS model. (b) A typical SAW on a $3 \times \infty$ strip of the square lattice, with periodic boundary conditions. The vertical bonds directly joining the top and bottom rows are not drawn. The walk shown belongs to the class $b_{N}$ as defined in the text.

following the path of a typical directed SAW, a sequence of spins is traced out. By construction, domains of parallel spins must be at least two lattice spacings in size. Consequently we have obtained one of the degenerate ground states of the RS model $(k=2)$. We can generalize this construction to arbitrary $k$ in a straightforward manner to SAWs in which there must be at least $k-1$ horizontal steps between vertical steps. These directed SAW configurations are isomorphic to the degenerate ground states of the Ising chain when $J_{k} / J_{1}=-1 / k$.

These results lead us to consider the problem of directed SAWs on lattice strips of arbitrary width $l$ (see Fig. 3b). In the infinite twodimensional limit $(l \rightarrow \infty)$, this has been solved exactly by Fisher and Sykes $^{(14)}$ in connection with obtaining rigorous bounds on the connective constant or effective coordination number for random SAWs on the square lattice. Our solution complements their work by finding the generating function for directed SAWs on finite strips of arbitrary width.

We consider here periodic boundary conditions in the transverse direction by wrapping the strip onto a cylinder. The free boundary case can also be treated by the method described here, but the details are considerably more complicated. We derive the generating function for the $l=3$ case explicitly, and generalization to arbitrary $l$ follows directly.

Let $a_{N}$ be the total number of $N$-step-directed SAWs. This number consists of all walks with the last step horizontal $\left(\equiv b_{N}\right)$, walks with the second-to-last step horizontal and the last step vertical $\left(\equiv c_{N}\right)$, and walks with the third-to-last step horizontal and the last two steps vertical ( $\left.\equiv d_{N}\right)$. Now $b_{N}$ can be obtained by adding a horizontal bond to any walk in the 
three classes. That is,

$$
b_{N}=b_{N-1}+c_{N-1}+d_{N-1}
$$

We obtain $c_{N}$ by adding a vertical bond in two possible directions to each walk in $b_{N-1}$ (this holds only for periodic boundaries). Similarly we obtain $d_{N}$ by adding a vertical bond to each walk in $c_{N-1}$. Thus

$$
c_{N}=2 b_{N-1} \text { and } d_{N}=c_{N-1}
$$

Combining these yields for the total number of $N$-step-directed SAWS,

$$
\begin{aligned}
a_{N} & =b_{N}+c_{N}+d_{N} \\
& =a_{N-1}+2 a_{N-2}+2 a_{N-3}
\end{aligned}
$$

For the case of a strip of $l$ lattice spacings we find similarly

$$
a_{N}=a_{N-1}+2\left(a_{N-2}+a_{N-3}+\cdots+a_{N-1}\right)
$$

In the limit $N \rightarrow \infty, a_{N} \sim \mu_{l}^{N}$, where the connective constant $\mu_{l}$ is the largest root of the characteristic equation derived from (4d), $x^{l}=x^{l-1}+$ $2 x^{l-2}+2 x^{l-3}+\cdots+2$, and this gives $\mu_{2}=2, \mu_{3}=2.2696 \ldots$, and $\mu_{4}$ $=2.3593 \ldots$, etc. As $l \rightarrow \infty, \mu_{l}$ approaches a finite limit which is the solution of $x^{l}=x^{l-1}\left(1+2 / x+2 / x^{2}+\cdots\right)$, or $x=(1+1 / x) /(1-$ $1 / x)$. From this we find that $\lim _{l \rightarrow \infty} \mu_{t}=1+\sqrt{2}=2.4142 \ldots$ In this limit our result duplicates that of Fisher and Sykes.

In summary, we have studied the properties of a finite onedimensional Ising chain with competing interactions $J_{1}$ and $J_{k}$. At zero temperature there exists an infinitely degenerate ground state for a particular value of $J_{k} / J_{1}$. We have calculated this degeneracy exactly for the infinite chain and for the finite chain with free boundary conditions. In the latter case the degeneracy can be expressed as the terms in a generalized Fibonacci sequence. We have also shown that the Ising chain is closely related to the following problems: the PUD model, a two-dimensional Ising system with a periodic arrangement of ferro- and antiferromagnetic bonds, close packing of dimers on a ladder, a $2 \times \infty$ strip of the square lattice, and directed self-avoiding walks on the ladder. Finally we have generalized, and calculated exactly, the properties of directed SAWs on lattice strips of arbitrary width.

\section{ACKNOWLEDGMENTS}

I am especially grateful to H. E. Stanley for proposing the approach for some of the derivations presented in this paper. I wish to thank him, and R. Bidaux, P. J. Reynolds, and T. Witten for many illuminating discussions. I also thank A. Huber and S. Muto for a critical reading of the manuscript and many constructive suggestions. 


\section{REFERENCES}

1. G. Toulouse, Commun. Phys. 2:115 (1977).

2. S. Kirkpatrick, Phys. Rev. B 16:4630 (1977).

3. S. Redner and H. E. Stanley, J. Phys. C 10:4765 (1977); S. Redner and H. E. Stanley, Phys. Rev. B 16:4901 (1977).

4. U. Enz, Physica (Utrecht) 26:698 (1960).

5. R. J. Elliott, Phys. Rev. 124:346 (1961).

6. R. M. Hornreich, M. Luban, and S. Shtrikman, Phys. Rev. Lett. 35:1678 (1975).

7. W. Selke, Z. Phys. B 29:133 (1978).

8. M. E. Fisher and W. Selke, Phys. Rev. Lett. 44:1502 (1980); M. E. Fisher and W. Selke, preprint.

9. Solitons and Condensed Matter Physics, A. R. Bishop and T. Schneider, eds. (Springer Verlag, Berlin, 1978), and references therein.

10. J. von Boehm and P. Bak, Phys. Rev. Lett. 42:122 (1979).

11. J. Stephenson, Can. J. Phys. 48:1724 (1970); R. M. Hornreich, R. Liebmann, H. G. Schuster, and W. Selke, Z. Phys. B 35:91 (1979).

12. G. André, R. Bidaux, J.-P. Carton, R. Conte, and L. deSeze, J. Phys. (Paris) 40:479 (1979).

13. J. Villain, J. Phys. C 10:1717 (1977).

14. M. E. Fisher and M. F. Sykes, Phys. Rev. 114:45 (1959). 